\title{
Atypical aortic coarctation as a cause of a cardiomyopathy
}

\author{
F. Alsemgeest · O. Kamp · C.B. Marcu
}

Published online: 2 June 2015

(C) The Author(s) 2015. This article is published with open access at Springerlink.com

\begin{abstract}
Atypical locations for aortic coarctation have been previously described. However, to our knowledge, no case has been described of a rapidly progressive dilated cardiomyopathy caused by an atypical coarctation, with a rapid normalisation of ventricular function after treatment.
\end{abstract}

Keywords Cardiomyopathy - Atypical aortic coarctation · MR $\cdot$ CT $\cdot$ Echocardiography $\cdot$ Hypertension $\cdot$ Aorta $\cdot$

Coarctation $\cdot$ Aortic coarctation $\cdot$ Pulmonary hypertension

A 40-year-old woman with a history of hypertension was referred to our hospital with progressive symptoms of exertional shortness of breath. On physical examination, her blood pressure was $140 / 90 \mathrm{mmHg}$ in the right arm and $120 / 85 \mathrm{mmHg}$ in the left arm. Auscultation of the heart revealed a grade III/VI loud systolic murmur, best heard in the suprasternal region and radiating to the back. Electrocardiogram and laboratory results were normal. Echocardiography showed a hypertrophic left ventricle with a poor left ventricular systolic function, restrictive diastolic function, moderate mitral regurgitation, pulmonary hypertension (estimated systolic pulmonary artery pressure (sPAP): $65 \mathrm{mmHg}$ ) and a dilated right ventricle. Continuous Doppler imaging of the proximal descending aorta showed a peak velocity of $4.75 \mathrm{~m} / \mathrm{s}$ (corresponding to a peak pressure gradient of $90 \mathrm{mmHg}$; Fig. 1a). Cardiac magnetic resonance imaging was performed and confirmed the diagnosis of aortic coarctation, but in a rather atypical location involv- ing the aortic arch (Fig. 1b). Computed tomography angiography furthermore revealed occluded left subclavian and left carotid arteries. Cardiac catheterisation showed normal coronary arteries and a mean pulmonary arterial pressure of $47 \mathrm{mmHg}$. The patient underwent stenting of the coarctation, which was unfortunately complicated by dissection of the descending thoracic aorta for which a conservative regimen was followed. One month after the procedure, the patient's symptoms rapidly disappeared and her left ventricular function normalised.

Open Access This article is distributed under the terms of the Creative Commons Attribution License which permits any use, distribution, and reproduction in any medium, provided the original author(s) and the source are credited.

F. Alsemgeest $(\bowtie) \cdot$ O. Kamp · C.B. Marcu

Department of Cardiology,

VU University Medical Center Amsterdam,

De Boelelaan 1117,

$1081 \mathrm{HZ}$, Amsterdam, The Netherlands

e-mail: ferryalsemgeest@gmail.com 
Fig. 1 Imaging of the atypical aortic coarctation. a Doppler imaging of the descending aorta showing an elevated peak pressure gradient $(90 \mathrm{mmHg})$. b Magnetic resonance and $\mathbf{c}, \mathbf{d}$ computed tomographic images showing a coarction aorta on an atypical location in the aortic arch (arrows)
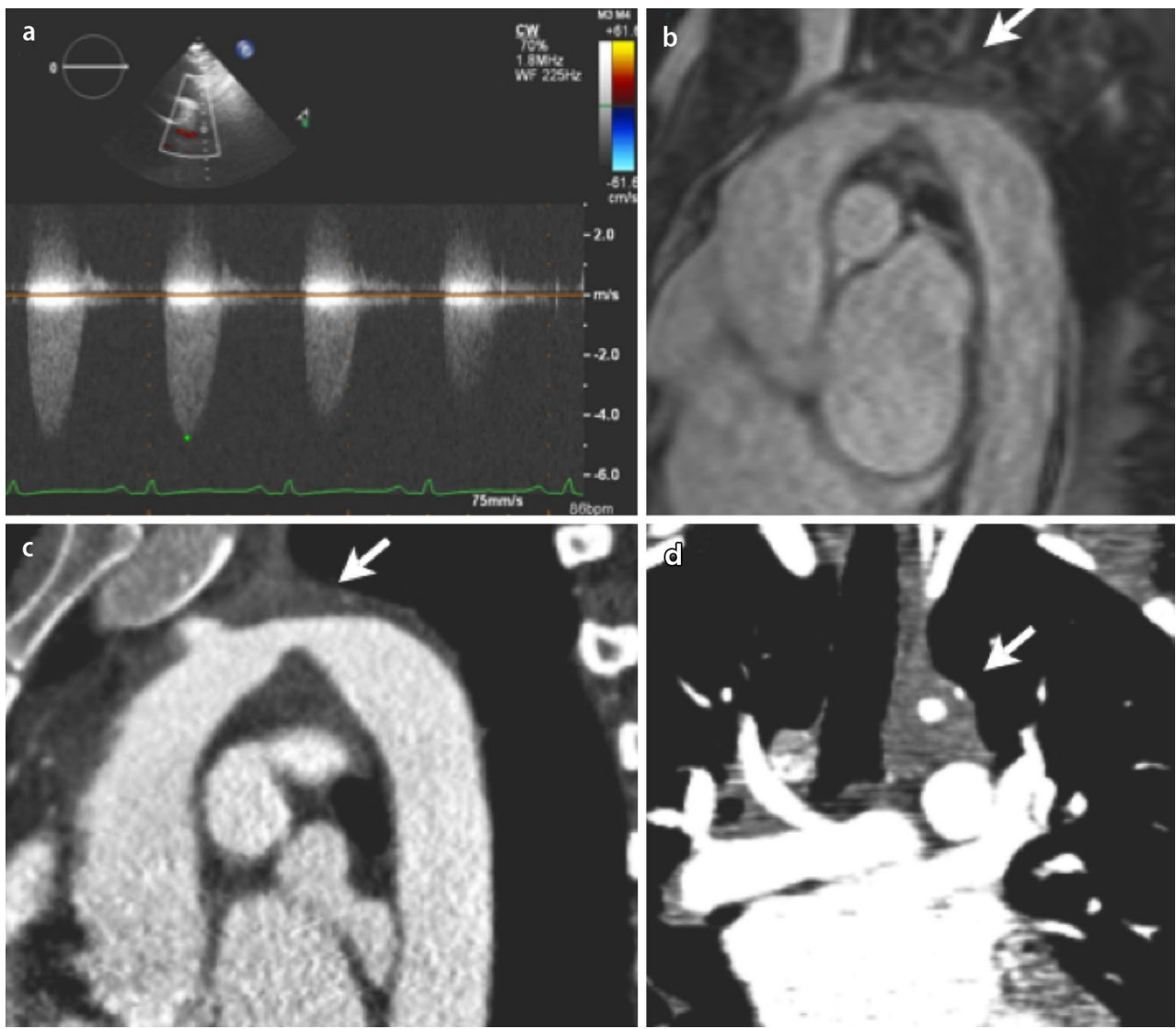